\title{
Analysis of membraneless fuel cell using laminar flow in a Y-shaped microchannel
}

\author{
Min-Hsing Chang ${ }^{\mathrm{a}, *}$, Falin Chen ${ }^{\mathrm{b}}$, Nai-Siang Fang ${ }^{\mathrm{b}}$ \\ a Department of Mechanical Engineering, Yung-Ta Institute of Technology and Commerce, \\ PingTung 909, Taiwan, Republic of China \\ b Institute of Applied Mechanics, National Taiwan University, Taipei 10764, Taiwan, Republic of China \\ Received 31 August 2005; received in revised form 3 November 2005; accepted 12 November 2005 \\ Available online 27 December 2005
}

\begin{abstract}
In this study, we implemented a theoretical analysis for a novel microfluidic fuel cell that utilizes the occurrence of laminar flows in a Y-shaped microchannel to keep the separation of fuel and oxidant streams without turbulent mixing. The liquid fuel and oxidant streams enter the system at different inlets, and then merge and flow in parallel to one another through the channel between two electrodes without the need of a membrane to separate both streams. A theoretical model containing the flow kinetics, species transport, and electrochemical reactions within the channel and the electrodes is developed with appropriate boundary conditions and solved by a commercial CFD package. The performance of this novel fuel cell is analyzed by a systematic study with respect to some important physical factors and the geometric effect of channel size. Results indicate that the performance is primarily dominated by the mass transport to the electrodes especially at the cathode and could be raised significantly by using a high aspect ratio of cross-sectional geometry.
\end{abstract}

(C) 2005 Elsevier B.V. All rights reserved.

Keywords: Membraneless fuel cell; Laminar flow; Microchannel

\section{Introduction}

A novel fuel cell technology based on the microfluidic device has received much attention recently due to its potentially wide applications in portable power sources for cell phones, laptop computers, and many specialized devices such as remote sensors, diagnostic tests, and global positioning systems [1-3]. Such micro-structured fuel cells are able to provide longer duration with lighter weight without refueling, and meet the growing demands of portable high energy density power sources. Ferrigno et al. [4] first described a small redox fuel cell utilizing a design as indicated in Fig. 1 to remove the membrane usually used to separate anodic and cathodic compartments. The cell uses the same species (vanadium) as oxidant and reductant and both liquid streams enter the Y-shaped microchannel at different inlets. By utilizing the occurrence of laminar flow at low Reynolds number [5], both streams flow parallel through

\footnotetext{
* Corresponding author. Tel.: +886 8 7233733; fax: +886 87215649 .

E-mail address: mhchang@mail.ytit.edu.tw (M.-H. Chang).
}

the channel without convective mixing. The diffusion along the interface between both streams is limited and hence there is no need of a membrane to separate them. Such a novel design avoids many issues in miniaturization of conventional proton exchange membrane fuel cells (PEMFCs) [6-9]. For example, the membrane can become dried out especially at higher operation temperature, the water management is difficult [10], and the problem of fuel crossover through the membrane causes a mixed potential to lower the fuel performance [11]. The microfluidic fuel cell apparently demonstrates several advantages over PEMFC: it eliminates ohmic losses and fouling problems due to the membrane, the fabrication and water management are simpler, and the fuel cell size can be significantly reduced. Moreover, the liquid fuels have higher energy densities in comparison with gaseous fuels which is particular important for portable power source applications [12].

Recently, many studies have employed the nature of laminar flow in developing microfuel cells. Choban et al. [1] considered a microfluidic fuel cell consisting of a Y-shaped channel by using formic acid as the fuel [13] and oxygen dissolved in sulfuric acid as the oxidant. Their experimental results suggest 


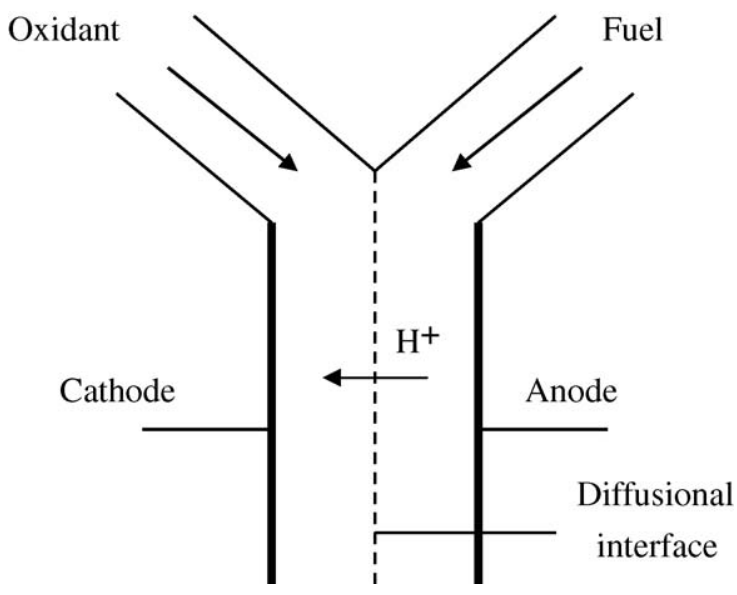

Fig. 1. Configuration of the membraneless microfuel cell in a Y-shaped microchannel.

that the transport of reactants through the concentration boundary layer to the electrodes restrains the fuel cell performance as well as the low concentration of oxidant in the cathode stream. Cohen et al. [2] demonstrated a new design for a planar membraneless microchannel fuel cell. Their planar design gives rise to large contact area between the electrodes and liquid streams, and can lead to higher power devices. Results for its power-producing capabilities were also discussed. Bazylak et al. [3] first presented a computational analysis of a membraneless microfuel cell constructed by a T-shaped microchannel configuration. They focused on how to improve fuel utilization while minimizing mixing across the fuel-oxidant interface. Their numerical simulations show that both microchannel and electrode geometry play important roles in system performance and propose that an extended tapered-electrode design can improve fuel utilization significantly. Hasegawa et al. [14] performed an experimental study for a direct hydrogen peroxide fuel cell and demonstrated that its power density is comparable to that of a typical air-breathing direct-methanol fuel cell with no expensive membrane-electrolyte assembly. Choban et al. [15] further studied the performance-limiting factors of membraneless microfuel cell and provided a full characterization of the properties through experimental measurements.

Since the performance of microfuel cell is dominated by the diffusion across the interface between the liquid streams and the depletion of reactants on the electrode surfaces from experimental observations, it is quite important to understand how the properties of both fuel and oxidant streams affect the overall cell performance. In this study, we present a numerical analysis of a membraneless fuel cell consisting of a Y-shaped microchannel in which fuel and oxidant flow in parallel at laminar regime. The system considered here is based on the design of Choban et al. [1] and a theoretical model is deduced to account for flow kinetics, species transport, and electrochemical reactions at the electrodes with appropriate boundary conditions. A detailed three-dimensional numerical simulation is performed to give physical insights for the characteristics of cell performance and provide a helpful guide to improve the design of such a microfuel cell. (a)
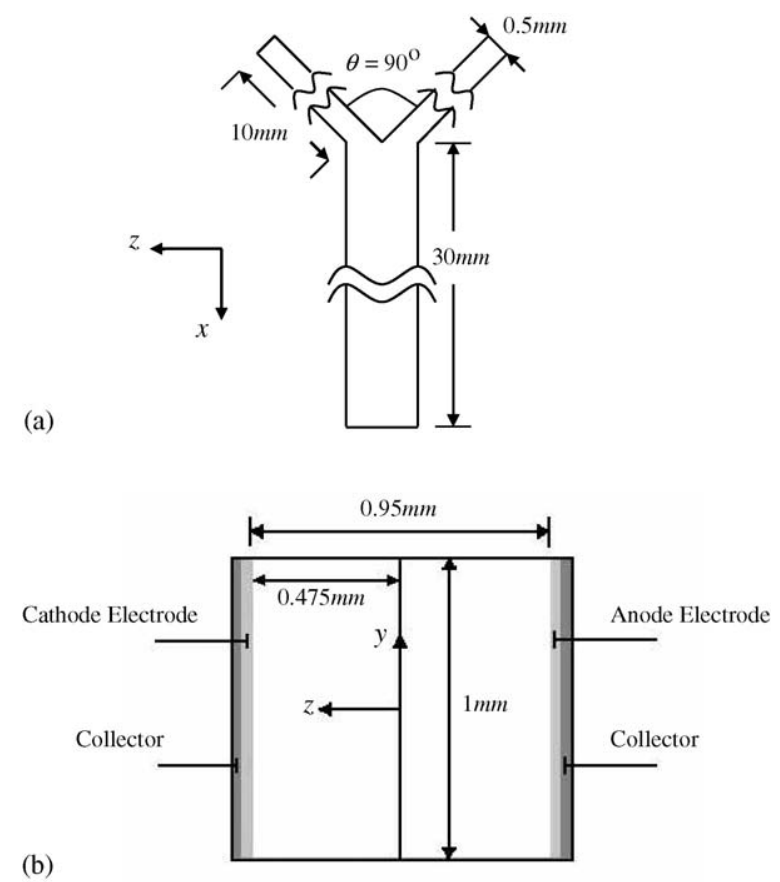

Fig. 2. Dimensions of the Y-shaped microchannel: (a) top view; (b) cross-section of main channel.

\section{Theoretical model and formulation}

We consider a microfluidic fuel cell in a Y-shaped microchannel as shown in Fig. 2. Formic acid is used as the fuel and aqueous solution of sulfuric acid saturated with oxygen as the oxidant. At the anode the formic acid releases energy, electrons and creates protons.

$\mathrm{HCOOH} \rightarrow \mathrm{CO}_{2}+2 \mathrm{H}^{+}+2 \mathrm{e}^{-}$

The electrons conduct to the cathode through external circuit and the protons diffuse to the oxidant flow across the interface between the liquid streams. At the cathode, the oxygen contained in the oxidant flow reacts with electrons taken from the electrode and protons from the sulfuric acid solution to form water.

$\mathrm{O}_{2}+4 \mathrm{H}^{+}+4 \mathrm{e}^{-} \rightarrow 2 \mathrm{H}_{2} \mathrm{O}$

Such a design of laminar flow-based fuel cell avoids the usage of membrane in conventional PEMFC as well as the accompanying problem of membrane drying out. The products of the overall reaction, carbon dioxide and water, can be removed automatically by the flowing streams since the carbon dioxide is highly soluble in water at room temperature. Assume the system is isothermal and both the fuel and oxidant are pressure-driven steady incompressible flows, the continuity and momentum equations are:

$\frac{\partial u_{i}}{\partial x_{i}}=0$,

$u_{j} u_{i, j}=-\frac{1}{\rho} \frac{\partial p}{\partial x_{i}}+v \Delta u_{i}$, 
where $p$ is the pressure, $\rho$ the density, $v$ the kinematic viscosity, and $\Delta$ the Laplacian operator. The concentration equation of species is defined by Fick's law [16]:

$u_{j} \frac{\partial C_{i}}{\partial x_{j}}=D_{i} \Delta C_{i}+\frac{e_{i}}{n F} S_{i}$,

where the subscript ' $i$ ' represents the reactant species, $C$ the concentration, $D$ the diffusion coefficient, $e$ the mole number, $n$ the number of electrons transferred in the reaction, $F$ the Faraday constant, and $S$ the rate of electrochemical reaction in Eqs. (1) and (2) expressed by the volumetric rate of current generation with unit $\mathrm{Am}^{-3}$. The electric potential equation in both anode and cathode streams can be written as:

$\sigma_{i} \Delta \Phi_{i}=S_{i}$

where $\sigma$ is the electric conductivity and $\Phi$ the electric potential. The reaction rate $S_{i}$ is given by using the Bulter-Volmer equation in the form:

$S_{i}=a j_{0}\left(\frac{C_{i}}{C_{i}^{0}}\right)^{\beta_{i}}\left[\exp \left(\frac{\alpha_{\mathrm{a}} n F}{R T} \eta\right)-\exp \left(-\frac{\alpha_{\mathrm{c}} n F}{R T} \eta\right)\right]$,

where $a$ is the density of the catalyzed active area on the electrode, $j_{0}$ the exchange current density at the reference reactant concentration $C_{i}^{0}, \beta_{i}$ the reaction order of species $i, \alpha_{\mathrm{a}}$ and $\alpha_{\mathrm{c}}$ are the charge transfer coefficient, respectively, for the anodic and cathodic reactions given in Eqs. (1) and (2), $R$ the universal gas constant, $T$ the absolute temperature, and $\eta$ the overpotential. It is assumed that the electrochemical reaction occurs at the anode and cathode only. Therefore, $S_{i}$ is equal to zero elsewhere in the system.

The boundary conditions at the inlets of the Y-shaped microchannel are given by the assigned values of velocity, pressure and concentration. At the exit, we assume:

$\frac{\partial u}{\partial x}=v=w=\frac{\partial C_{i}}{\partial x}=0$,

and at the interface between the electrode and the current collector, we have:

$u=v=w=\frac{\partial C_{i}}{\partial z}=0$.

At the liquid-liquid and liquid-electrode interfaces, the velocity, shear stress, concentration, and concentration flux are continuous across the interface. Moreover, the electric potential after the merging point of both streams is assumed to be continuous and satisfies the condition.

$\frac{\partial \Phi}{\partial x}=0$.

Once the governing equations are solved together with the boundary conditions, the current density $\vec{j}$ can be determined by:

$j_{i}=-\sigma \frac{\partial \Phi}{\partial x_{i}}$

Note that only the $z$-component normal to the electrode surface is useful and contributes to the power output of fuel cell.
The commercial CFD package, CFDRC, is used to perform the numerical simulation. The governing equations are discretized and solved by the finite volume method with structured computational grid technique. The geometric dimensions of the Y-shaped microchannel are demonstrated in Fig. 2. To ensure the accuracy of numerical results, we have made the grid tests and results indicate that the grid numbers $174(56) \times 23 \times 15$ in $x, y$, and $z$ directions, respectively, are sufficient to yield converged results with economic consumption of CPU time, where the number in the bracket is the number of grids along the branch of the Y-shaped microchannel.

\section{Results and discussion}

We consider the microfuel cell operates at room temperature $T=298 \mathrm{~K}$ and the corresponding reversible open circuit voltage $E_{\mathrm{rev}}^{0}$ is $1.48 \mathrm{~V}[1,17]$. Some typical parameters used in anodic and cathodic flows are list in Table 1 . It has been found that in multi-stream laminar flow, the diffusion transverse to the flow direction causes the mixing of the two streams. The degree of mixing depends on the value of Peclet number defined by $P e=U H / D[1,5]$, where $U$ is the mean velocity and $H$ the channel height. This number represents the ratio of convective to diffusive transport. The variations of the mass fraction profiles along the microchannel with $P e$ are shown in Figs. 3 and 4 for formic acid and oxygen, respectively. At $P e=10^{3}$ as shown in Fig. 3(a), it is found that the molecules of formic acid diffuse into the cathodic flow and the diffusion zone extends gradually after the joint point of both streams. The profile of diffusion zone is wider near the top and bottom walls with an hourglass shape because the mixing is more pronounced near the walls due to slower flow velocity arising from no slip boundary condition. The width of diffusion zone $\Delta z$ at the wall is also in agreement with the results of scale analysis [5] expressed in the form:

$\Delta z \propto\left(\frac{D H x}{U}\right)^{1 / 3}$

It is noticed that the diffusion zone almost occupies half the profile area of cathodic channel at the exit $x=30 \mathrm{~mm}$ and reaches the cathode at top and bottom walls. Thus, fuel crossover may occur in this case since both streams have more time to be in diffusion contact at lower Peclet number resulting in the enhancement of mixing. When $P e$ is increased to $10^{4}$ as shown in Fig. 3(b), the width of diffusion zone reduces obviously and becomes even thinner at $P e=10^{5}$ as shown in Fig. 3(c). Therefore, the laminar flow fuel cell should operate at a high Peclet number to avoid mixed potential at the cathode due to fuel

Table 1

Some parameters used in anodic and cathodic flows

\begin{tabular}{llc}
\hline & Anodic flow & Cathode flow \\
\hline Electrical conductivity, $\sigma\left(\mathrm{mho} \mathrm{m}^{-1}\right)$ & 11.47 & 43.00 \\
Exchange current density, $a j_{0}\left(\mathrm{~A} \mathrm{~m}^{-3}\right)$ & $3.82 \times 105$ & 100 \\
Charge transfer coefficient, $\alpha$ & 0.5 & 0.5 \\
Reaction order, $\beta$ & 1 & 1 \\
Concentration $(\mathrm{M})$ & 2.1 & 0.5 \\
\hline
\end{tabular}




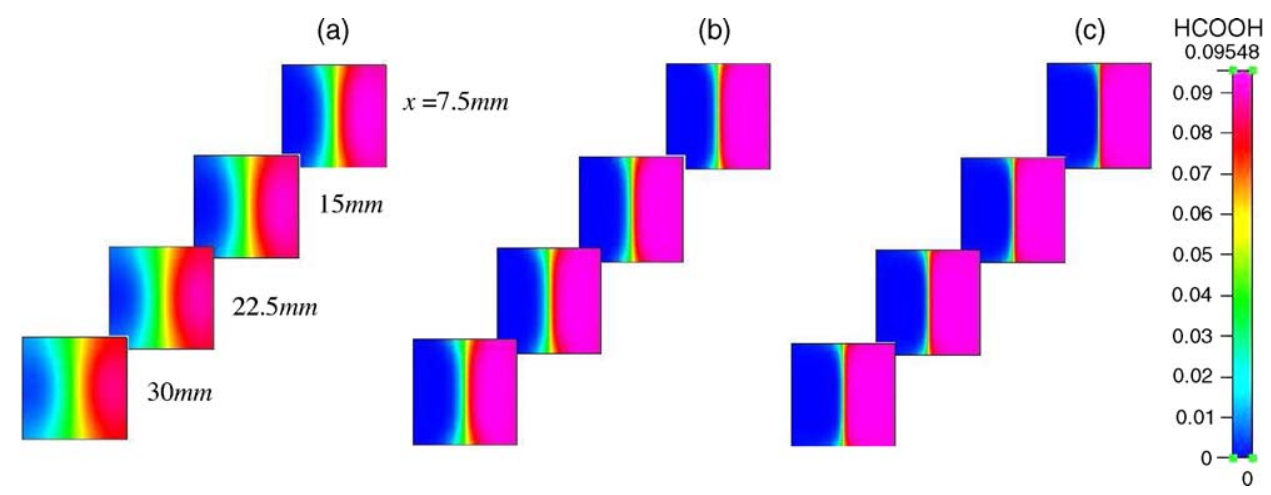

Fig. 3. The variations of mass fraction contour plot of formic acid with three assigned values of Peclet number at four locations of $Y-Z$ cross-section along the microchannel: (a) $\mathrm{Pe}=10^{3}$; (b) $\mathrm{Pe}=10^{4}$; (c) $\mathrm{Pe}=10^{4}$.

crossover. These results theoretically verify the suggestions of previous experimental studies [1,5]. However, if the Peclet number is raised by increasing the mean velocity $U$, the flow rate is increased and the streams may deviate from steady state. The onset of hydrodynamics instability has been observed in experiments [1] and affects the performance of the membraneless fuel cell significantly. It would be important to find the stability boundary and investigate the instability mechanism for this system to determine the optimum flow rate.

Similar results for diffusion zone also can be observed in the variations of concentration profile of oxygen with respect to $P e$ as shown in Fig. 4(a)-(c). Note that the concentration boundary layer adjacent to the surface of cathode is quite apparent at $P e=10^{3}$ and grows gradually down the stream as demonstrated in Fig. 4(a). This wide concentration boundary layer is due to the low oxidant concentration. Since low Peclet number indicates slow flow velocity, the oxidant stream has more time to contact and react with the electrode and most of oxygen near the electrode is consumed to form the concentration boundary layer. The low diffusivity and concentration make the concentration flux toward the surface of cathode be small and thus the thickness of concentration boundary layer grows along the channel. As $P e$ increases to $10^{4}$ as shown in Fig. 4(b), the thickness of concentration boundary layer reduces obviously and still becomes thinner at $P e=10^{5}$ in Fig. 4(c). The thinner concentration boundary layer causes a steep concentration gradient and enhances the transport of reactant through the layer to the electrode signifi- cantly. In comparison with the results of Fig. 3(a)-(c), one can see that the concentration boundary layer of formic acid solution at anode is quite small even at $P e=10^{3}$. The reason is that the concentration of formic acid is greatly higher than that oxygen as given in Table 1. Hence, the high concentration of anodic stream improves the corresponding concentration flux and causes the concentration boundary layer to be negligible.

Fig. 5(a) and (b) illustrate the performance of this membraneless fuel cell for the three typical cases of $\mathrm{Pe}$ considered in Figs. 3 and 4 via the plots of output potential and corresponding power density against current density, respectively. As shown in Fig. 5(a), the slope of each curve is similar to that of a typical fuel cell with activation and concentration losses. Obviously, the cell performance is better at higher value of $\mathrm{Pe}$ since the curve falls more slowly with increase of current density. However, the maximum Peclet number is limited by the possible onset of hydrodynamics instability. When the current density increases from zero, the voltage drop is primarily due to the activation losses which are caused by the electrochemical reactions taking place on the surface of electrode. A proportion of cell voltage is lost in transferring the electrons to or from the electrode. Following the increase of current density, the concentration losses that results from the change in concentration of the reactants at the surfaces of the electrodes gradually becomes dominant. Once the reduction in concentration fails to transport sufficient reactant to the electrode surface, the output potential will fall rapidly with a slight increase of current density. In Fig. 5(b), we

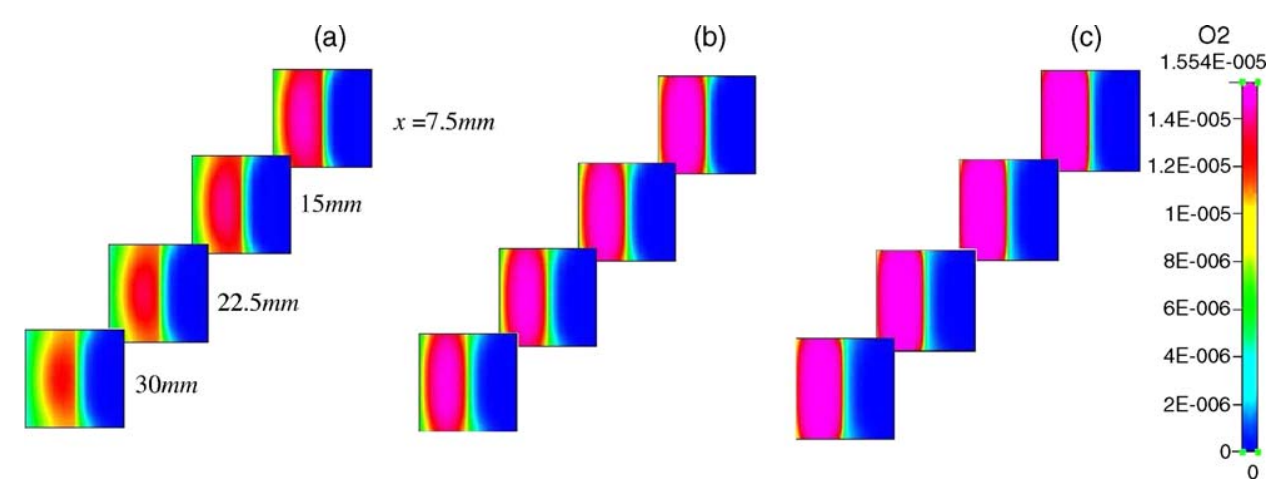

Fig. 4. The variations of mass fraction contour plot of oxygen with three assigned values of Peclet number at four locations of $Y-Z$ cross-section along the microchannel: (a) $P e=10^{3}$; (b) $P e=10^{4}$; (c) $P e=10^{4}$. 

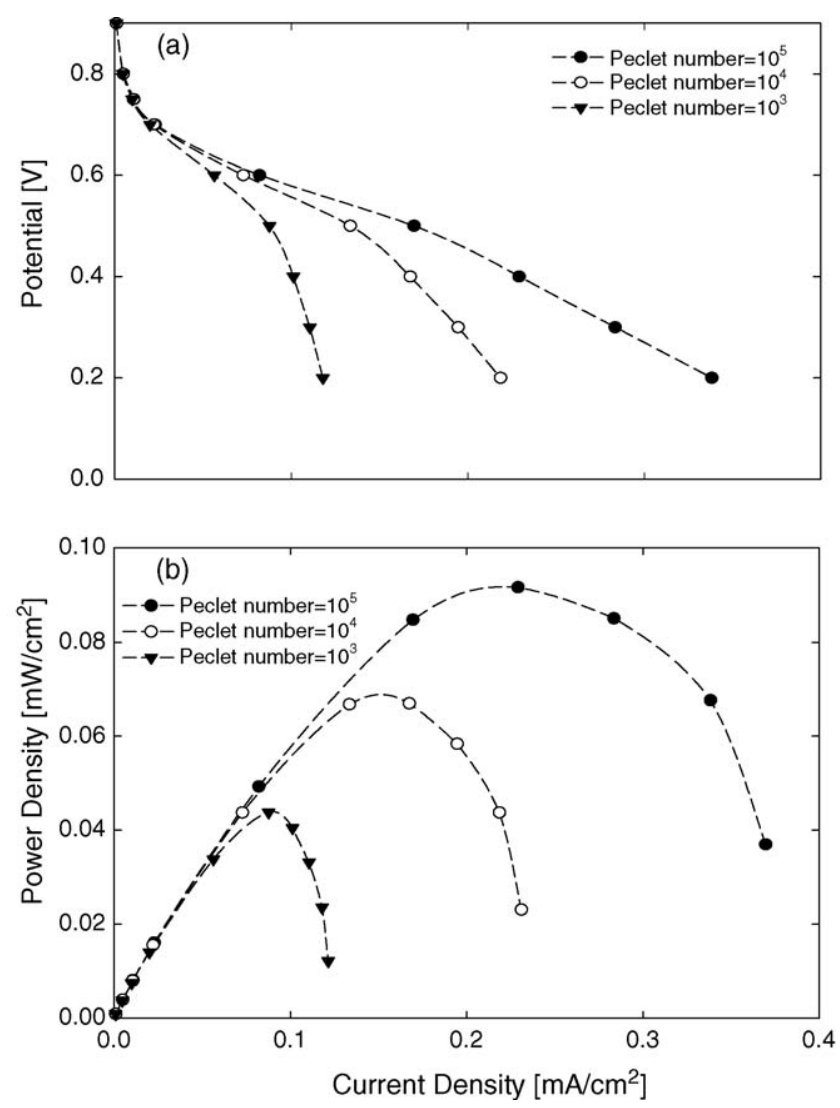

Fig. 5. Variations of cell performance with three assigned values of Peclet number: (a) the load curves; (b) the corresponding power density curves.

observe that the power density for each case rises first and them reaches a maximum. Afterward the potential drop due to concentration losses increases significantly and causes the power density to drop first as well. To compare the present numerical results with those of experimental work [1], it is found that the predicted potential for an assigned value of current density in this study is always less than that of experimental measurement. Since some parameters used in the theoretical model are not given in the work [1], it is somewhat difficult to make an exactly quantitative comparison between the present theoretical predictions with the experiments. Nevertheless, the qualitative behaviors of cell performance in both studies are still in good agreement.

The results displayed in Fig. 5 can also be inferred from the contour plots of mass fracture of oxygen as shown in Fig. 4. In Fig. 4(a), the concentration boundary layer is relatively thicker at $P e=10^{3}$. Thus, the concentration loss is more pronounced in this case that causes the poor cell performance as indicated in Fig. 5. The thickness of concentration boundary layer decreases with $P e$ gradually, so the cell performance is raised simultaneously as demonstrated in Fig. 5 for the cases $P e=10^{4}$ and $10^{5}$. As the concentration boundary layer is insignificant near the surface of anode, it can be proposed that the performance of such a membraneless fuel cell is limited by the cathode. The data of the exchange current density for anode and cathode given in Table 1 also indicates that the activation losses are primarily due to the
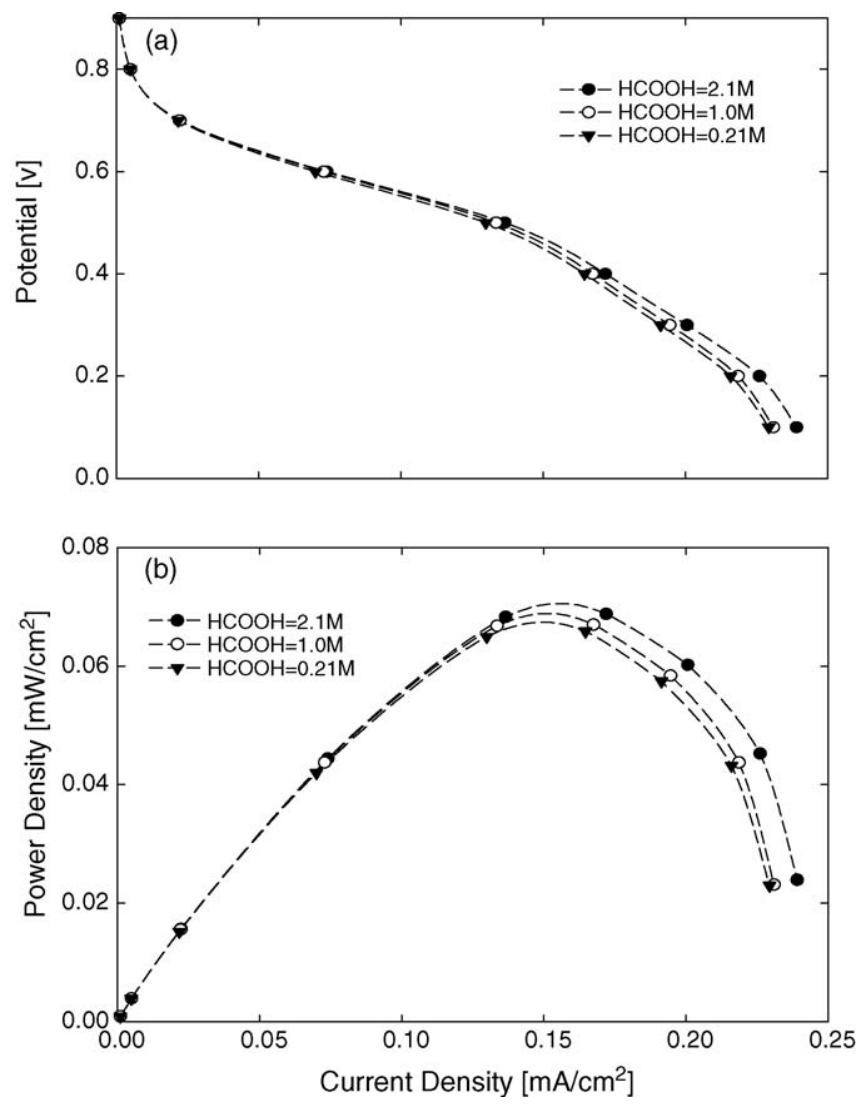

Fig. 6. Variations of cell performance with three assigned concentrations of formic acid solution: (a) the load curves; (b) the corresponding power density curves.

lower chemical activity at cathode. Accordingly, we may conclude that the cell performance well be enhanced profoundly if the concentration of oxygen in cathodic stream can be increased to raise concentration flux or the exchange current density at the cathode can be made as high as possible. While a variation of concentration for formic acid solution will produce small influence on cell performance only. These inferences are examined in Figs. 6 and 7 by changing the concentrations of reactants in anodic and cathodic streams, respectively. As shown in Fig. 6(a) and (b), three typical concentrations of formic acid solution, $2.1 \mathrm{M}, 1 \mathrm{M}$, and $0.21 \mathrm{M}$ are considered. Note that the case $2.1 \mathrm{M}$ is the same as the case $P e=10^{4}$ in Fig. 5. It is found that if the fuel concentration reduces to $1 \mathrm{M}$ or even smaller $0.21 \mathrm{M}$, the performance still does not drop significantly. These results are consistent with the experimental findings [1]. While if we can raise the concentration of oxygen in cathodic stream, the cell performance appears to be improved significantly as shown in Fig. 7(a) and (b), in which three assigned values of oxygen concentration $0.5 \mathrm{mM}, 1 \mathrm{mM}$, and $5 \mathrm{mM}$ are investigated. One can see that if the concentration of oxygen is 10 times larger, the current density is also increased approximately ten times. Thus, the present analyses confirm the experimental results that this membraneless microfuel cell is cathodic limited, and suggest that it is a crucial factor in improving cell performance to increase the concentration of oxygen in the oxidant stream. 

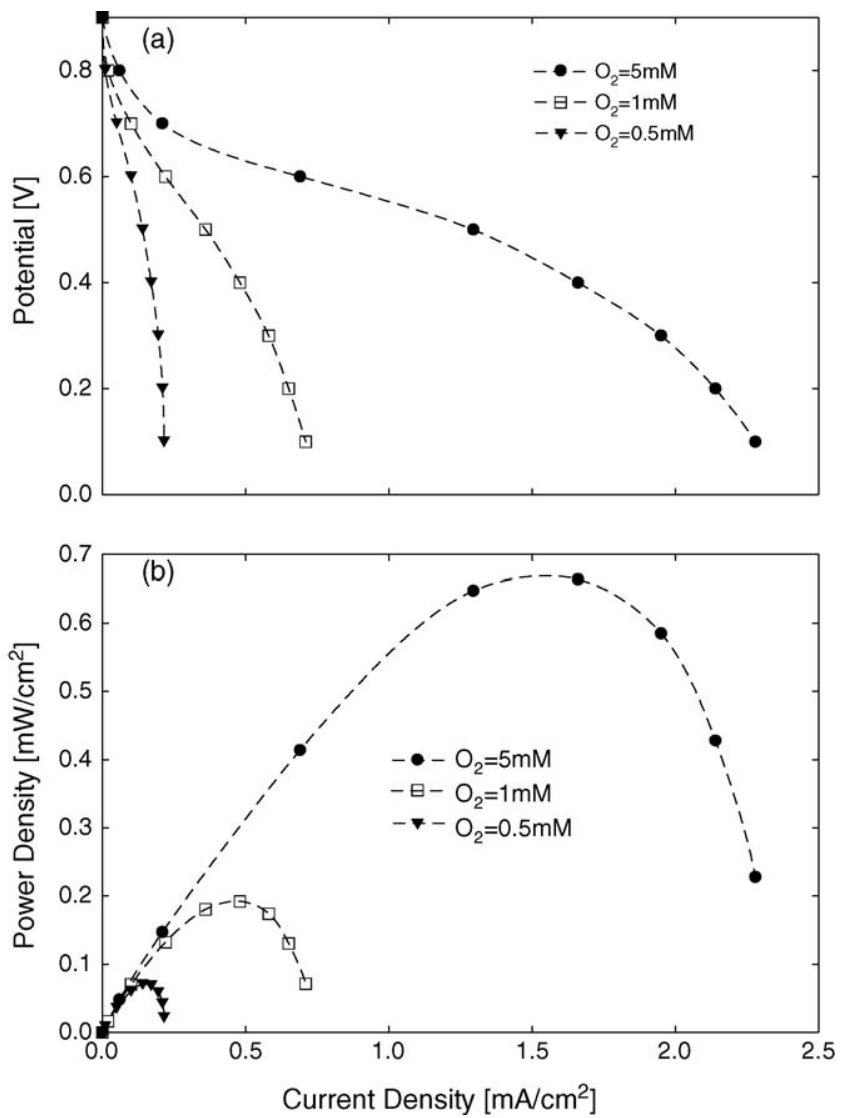

Fig. 7. Variations of cell performance with three assigned concentrations of oxygen in sulfuric acid solution: (a) the load curves; (b) the corresponding power density curves.

The geometry of the cross-section of the microchannel is also an important factor to influence the cell performance. Fig. 8 illustrates the variations of current density with volumetric flow rate for three assigned square cross-sectional area. For a given volumetric flow rate, it is found that a reduction of cross-sectional area increases the current density significantly. Since the mean flow velocity will be increased by the reduc-

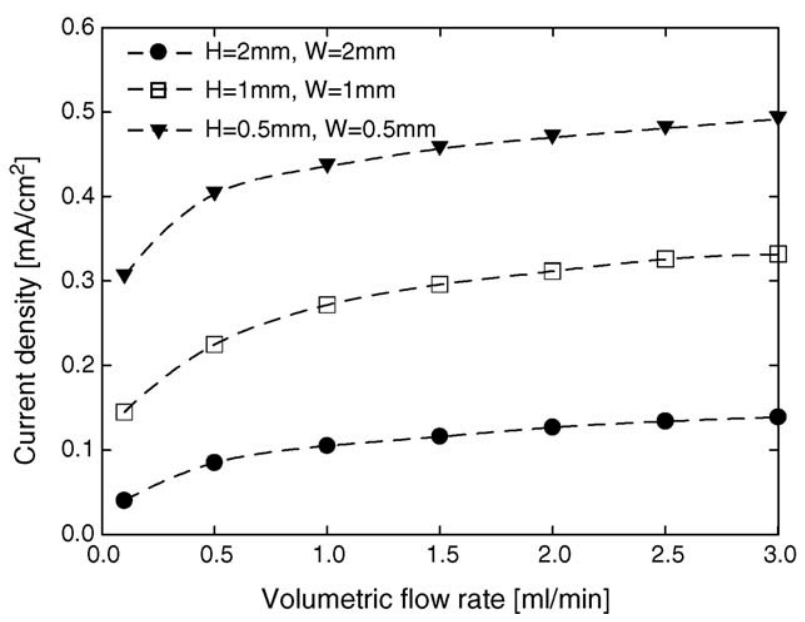

Fig. 8. Variations of current density against volumetric flow rate for three different square channel sizes.
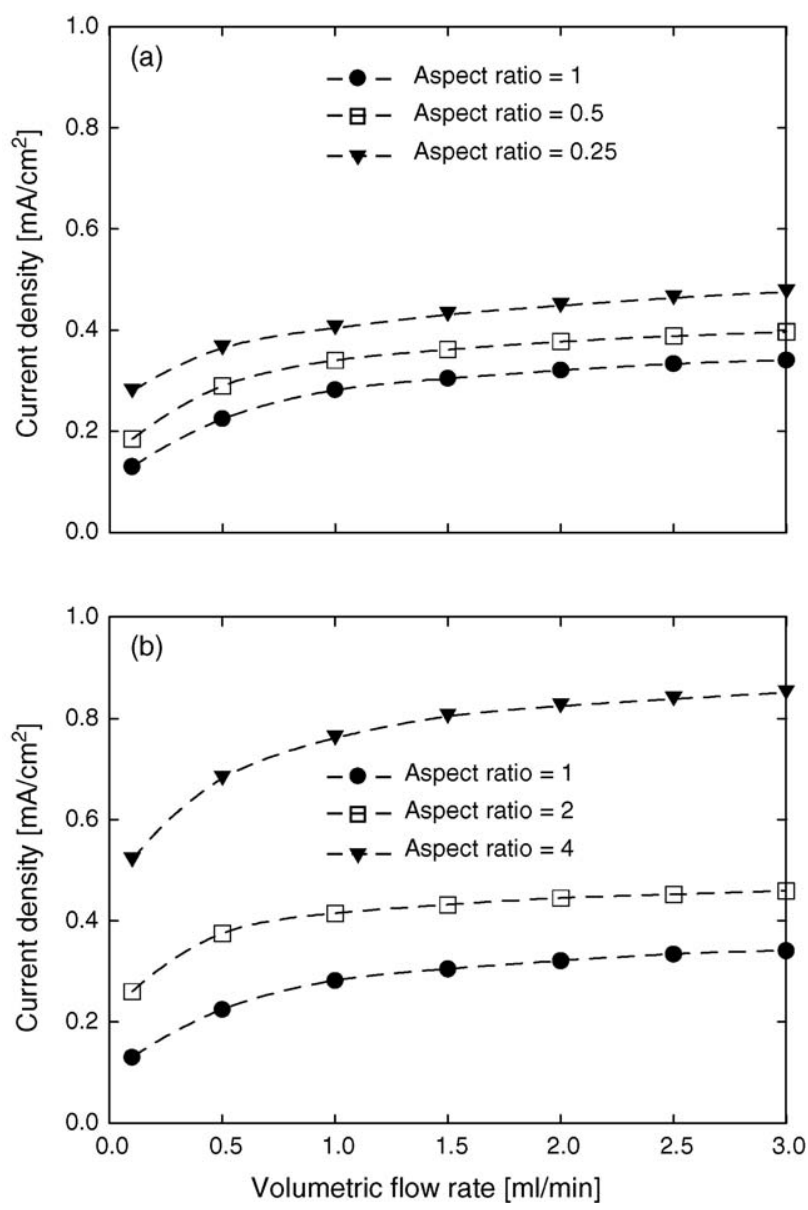

Fig. 9. Variations of current density against volumetric flow rate for three assigned values of aspect ratio: (a) the channel width is fixed at $W=1 \mathrm{~mm}$; (b) the channel height is fixed at $H=1 \mathrm{~mm}$.

tion of cross-sectional area under the same volumetric flow rate, the Peclet number becomes larger that causes the concentration boundary layer to be thinner. Consequently, the resistance of mass transport to the electrodes reduces and the more intense concentration flux raises the cell performance, especially at the cathode. These simulations are also in agreement with the experimental results [1]. In this study, we further investigate the effect of cross-sectional aspect ratio defined by the ratio of the channel height $H$ to the channel width $W$. The performance for several assigned values of aspect ratio against volumetric flow rate is demonstrated in Fig. 9(a) and (b). In Fig. 9(a), the channel width is fixed at $W=1 \mathrm{~mm}$. Therefore, a smaller aspect ratio also indicates smaller cross-sectional area. If the volumetric flow rate keeps constant, a smaller aspect ratio will give better performance due to the same reason as we have discussed. However, if we adjust the aspect ratio in this way, the improvement of cell performance is quite limited. While if we change the aspect ratio with a constant channel height $H=1 \mathrm{~mm}$, the cross-sectional area decreases with higher aspect ratio and the cell performance rises more rapidly in this situation. It is noted that the case with aspect ratio 0.25 in Fig. 9(a) has the same cross-sectional area as the case with aspect ratio 4 in Fig. 9(b). Comparing both cases one can see that the performance will be 
better if the geometry of cross-section has higher aspect ratio for a fixed cross-sectional area since the Peclet number will be larger in such a geometric shape, although it is restrained by the diffusion zone between anodic and cathodic streams. The results in Figs. 8 and 9 also reveal that the effect of volumetric flow rate on cell performance is significant when it is less than $1 \mathrm{ml} \mathrm{min}{ }^{-1}$ for any cross-sectional size. While if the volumetric flow rate is greater than this value, an increase of flow rate only produces little influence and the cell performance tends to a constant eventually.

\section{Conclusions}

We have conducted a numerical analysis to study the performance of a laminar flow-based membraneless microfuel cell consisting of a Y-shaped microchannel. A theoretical model is developed to simulate the flow field, mass transport, and electrochemical reactions within this system. Results show that it would produce better performance to operate the cell at higher Peclet number, and the fuel crossover due to the mixing between the fuel and oxidant streams can also be prevented. The performance of this membraneless microfuel cell is mainly restricted by the low concentration of oxygen in cathodic stream and the weak chemical activity on the surface of cathode. Thus, it would be helpful to enhance the electrocatalytic activity on cathode surface to raise cell performance. An increase of oxygen concentration will simultaneously improve the concentration flux to the electrode and produce a significant effect on the enhancement of performance.

The geometry of channel profile also plays an important role in the factors to affect the cell performance. For a fixed aspect ratio and volumetric flow rate, a reduction of cross-sectional area will give higher performance. While for a constant crosssectional area, a larger aspect ratio is preferred.

The present results have confirmed some predictions based on experimental measurements [1], and provide further theoretical insights which will benefit the design and development of such a membraneless microfuel cell. It will be interesting for future work to focus on the different fuel/oxidant combinations in order to compare their performance to the present study, and help the progress to make this novel membraneless microfuel cell be practically applied in portable power sources.

\section{Acknowledgements}

The financial support for this research from National Science Council of Taiwan through the grants NSC 94-2212-E-132005, NSC 94-2212-E-002-008, and NSC 93-2212-E-002-029 are gratefully acknowledged.

\section{References}

[1] E.R. Choban, L.J. Markoski, A. Wieckowski, P.J.A. Kenis, J. Power Sources 128 (2004) 54-60.

[2] J.L. Cohen, D.A. Westly, A. Pechenik, H.D. Abruna, J. Power Sources 139 (2005) 96-105.

[3] A. Bazylak, D. Sinton, N. Djilali, J. Power Sources 143 (2005) 57-66.

[4] R. Ferrigno, A.D. Stroock, T.D. Clark, M. Mayer, G.M. Whitesides, J. Am. Chem. Soc. 124 (2002) 12930-12931.

[5] R.F. Ismagilov, A.D. Stroock, P.J.A. Kenis, G. Whitesides, H.A. Stone, Appl. Phys. Lett. 76 (2000) 2376-2378.

[6] J. Yu, P. Cheng, Z. Ma, B. Yi, Electrochim. Acta 48 (2003) 1537 1541.

[7] S.J. Lee, A.C. Chien, S.W. Cha, R. O'Hayre, Y.I. Park, Y. Saito, F.B. Prinz, J. Power Sources 112 (2002) 410-418.

[8] J. Yu, P. Cheng, Z. Ma, B. Yi, J. Power Sources 124 (2003) 40-46.

[9] J. Yeom, G.Z. Mozsgai, B.R. Flachsbart, E.R. Choban, A. Asthana, Sens. Actuators B 107 (2005) 882-891.

[10] J. Larminie, A. Dicks, Fuel Cell Systems Explained, 2nd ed., John Wiley, Chichester, 2003.

[11] M. Eikerling, A.A. Kornyshev, A.M. Kuznestov, J. Ulstrup, S. Walbran, J. Phys. Chem. B 105 (2001) 3646-3662.

[12] C.K. Dyer, J. Power Sources 106 (2002) 31-34.

[13] C. Rice, S. Ha, R.I. Masel, P. Waszczuk, A. Wieckowski, T. Barnard, J. Power Sources 111 (2002) 83-89.

[14] S. Hasegawa, K. Shimotani, K. Kishi, H. Watanabe, Electrochem. SolidState Lett. 8 (2005) A119-A121.

[15] E.R. Choban, P. Waszczuk, P.J. Kenis, Electrochem. Solid-State Lett. 8 (2005) A348-A352.

[16] R.B. Bird, W.E. Stewart, E.N. Lightfoot, Transport Phenomena, 2nd ed., John Wiley, New York, 2002.

[17] M. Weber, J.T. Wang, S. Wasmus, R.F. Savinell, J. Electrochem. Soc. 143 (1996) 158-160. 